\title{
Radiological and clinical results following high- dose intensity-modulated radiotherapy in recurrent craniopharyngioma: A case report
}

\author{
ANTONIO PIERRO ${ }^{*}$, SAVINO CILLA ${ }^{2 *}$, VINCENZO PICARDI $^{3}$, MARICA FERRO $^{3}$, GABRIELLA MACCHIA $^{3}$, \\ FRANCESCO DEODATO $^{3}$, MILLY BUWENGE ${ }^{3}$, GIUSEPPINA SALLUSTIO ${ }^{1}$ and ALESSIO G. MORGANTI ${ }^{3-5}$ \\ ${ }^{1}$ Radiology Unit, ${ }^{2}$ Medical Physics Unit, ${ }^{3}$ Radiation Oncology Unit and ${ }^{4}$ General Oncology Unit, \\ Fondazione di Ricerca e Cura 'Giovanni Paolo II', Università Cattolica del Sacro Cuore, Campobasso 86100; \\ ${ }^{5}$ Radiation Oncology Unit, Department of Experimental, Diagnostic and Specialty Medicine, \\ DIMES University of Bologna, S.Orsola-Malpighi Hospital, Bologna 40138, Italy
}

Received October 2, 2014; Accepted May 1, 2015

DOI: $10.3892 / \mathrm{ol} .2015 .3514$

\begin{abstract}
Craniopharyngiomas (CPs) are rare benign suprasellar tumors. The standard treatment for $\mathrm{CP}$ is complete surgical resection or partial resection followed by adjuvant radiotherapy (RT). Adjuvant RT is typically administered at a total dose of $54 \mathrm{~Gy}$ with $1.8 \mathrm{~Gy} /$ fraction. The current study reported the case of a young patient affected by recurrent craniopharyngioma, who was treated with irradiation subsequent to several surgical resections. Image fusion and intensity-modulated radiation therapy techniques were employed to deliver a high total dose (63 Gy with $2.1 \mathrm{~Gy} / \mathrm{frac}$ tion) with no severe acute toxicities recorded. At the 6-year follow-up, no radiological or clinical signs of disease progression or late sequelae were observed.
\end{abstract}

\section{Introduction}

Craniopharyngiomas (CPs) are rare benign suprasellar tumors that have an incidence of between 1.2 and 4.6\% (1-5) with no differences between males and females as well Caucasians and African Americans and with a bimodal age distribution, one peak occurring in children and the other in adults between the 4 th and 6 th decades of life (1-5). The survival rate at 5 years is $\sim 80 \%$ overall, with survival much better among children than the elderly (1-5). CPs arise from epithelial remnants of

Correspondence to: Dr Gabriella Macchia, Radiation Oncology Unit, Fondazione di Ricerca e Cura 'Giovanni Paolo II', Università Cattolica del Sacro Cuore, 1 Largo Agostino Gemelli, Campobasso 86100, Italy

E-mail: gmacchia@rm.unicatt.it

${ }^{*}$ Contributed equally

Key words: craniopharyngioma, pediatric, intensity-modulated radiation therapy, case report the embryonic Rathke's pouch, and is characterized by slow growth (2-5). CPs are divided into two histological subtypes: (i) Adamantinomatous CP, typically occurring in children and adolescents; and (ii) papillary $\mathrm{CP}$, observed almost exclusively in adults (2). Cystic and solid components with frequent calcifications characterize the adamantinomatous subtype, while the papillary subtype is commonly solid with low incidence of calcifications (2). In the adamantinomatous variant, the solid component frequently extends into the sellar region of the sphenoid bone, while the associated cystic component is situated outside and above the sella turcica (1).

The standard treatment for $\mathrm{CP}$ is complete surgical resection, or partial resection followed by adjuvant radiotherapy (RT) (3). The latter option has the advantage of reducing the risk of neurological, visual and endocrine (panhypopituitarism) complications (3). Adjuvant RT is typically administered at a total dose of $54 \mathrm{~Gy}$ with $1.8 \mathrm{~Gy} /$ fraction, although doses up to 59.4 Gy have previously been administered by fractionated proton RT (2). In order to improve the therapeutic outcomes, novel RT techniques have been proposed, including stereotactic radiosurgery (SRS), stereotactic RT (SRT) and intensity-modulated radiation therapy (IMRT) (3).

The treatment of relapse following surgery is complex and, in such cases, it is difficult to treat the disease without producing significant side effects, including optic neuropathy, neuropsychological sequel and delayed hypothalamic-pituitary damage (5). The aim of the current report was to present a case of CP treated with high-dose IMRT and subsequently monitored by clinical and instrumental evaluation, including magnetic resonance imaging (MRI).

\section{Case report}

A 7-year-old girl was diagnosed with cystic CP in March 1992, following presentation of symptoms including growth arrest, headache and panhypopituitarism, in addition to bitemporal hemianopia. Partial resection of the tumor and drainage into the cystic lesion were performed by craniotomy, followed by intracystic bleomycin administration (6). After 7 years, 


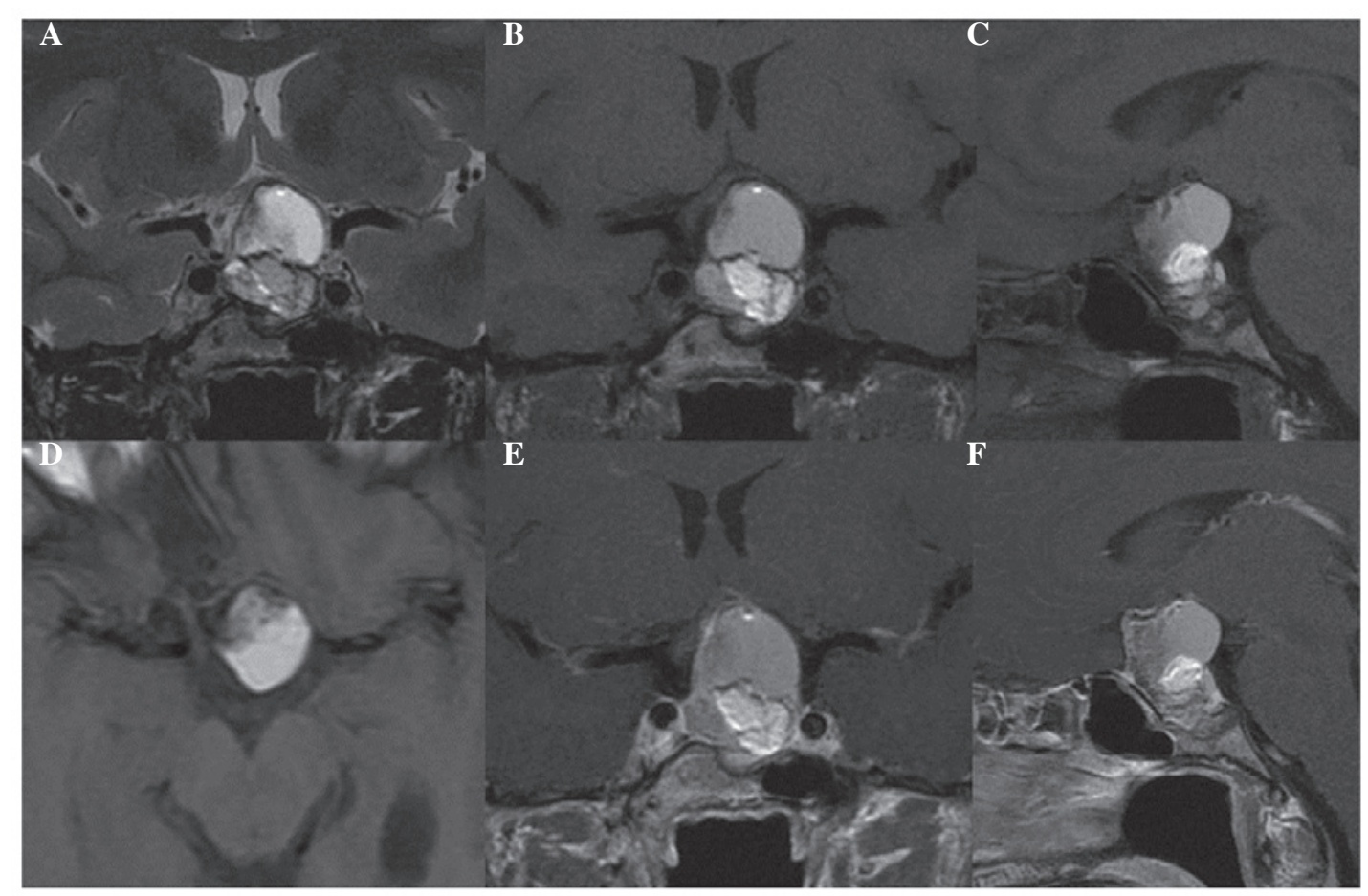

Figure 1. Magnetic resonance images prior to radiotherapy. (A) T2-weighted image, demonstrating the cystic and solid component of the mass in the sellar and suprasellar region. The T1-weighted (B) coronal, (C) sagittal and (D) axial images displaying the typical signal hyperintensity of the cystic components. (E) coronal and (F) sagittal T1-weighted images following infusion of paramagnetic contrast agent demonstrate the impregnation of the solid component.

in September 1999, an MRI examination documented local progression of the residual lesion. A further partial resection was performed by craniotomy. In March 2008, at 22 years of age, a local progression of the disease with bitemporal hemianopia was observed. A third partial resection was performed by trans-sphenoidal access. However, 2 months later (May 2008), the patient was referred to our RT unit reporting headache and fatigue. The patient was receiving regular replacement therapy with levothyroxine (100 mg/day), desmopressin acetate, estradiol, medroxyprogesterone acetate and somatropin. A neurosurgeon clinically diagnosed a fourth recurrence. Given the limited prospects of further salvage surgery, a novel potentially curative treatment with high-dose step-and-shoot intensity-modulated radiotherapy (IMRT) was prescribed. IMRT by which the intensity or radiant fluence of each beam is purposely altered by the summation of hundreds of beamlets, may represent a valid strategy in order to satisfy clinical goals of target and normal tissue doses: IMRT has been demonstrated to reduce the incidence of acute and late cerebral toxicities by assisting the conformality of dose distribution, confining the high-dose portions of radiation fields and reducing the absorbed dose and volume in critical organs (7). A total of 63 Gy in 30 fractions, 5 days per week were prescribed. Written informed consent was obtained from the patient and her family.

A brain MRI scan was performed utilizing a Signa Excite MR 1.5T system (GE Medical Systems, Milwaukee, WI, USA). Study of the whole brain was conducted with 5-mm slices using the following sequences: Fluid-attenuated inversion recovery (FLAIR), gradient echo (GRE) sequence, diffusion weighted imaging (DWI) and fast spin-echo (FSE) T1- and T2-weighted imaging. Following infusion of a paramagnetic contrast agent
(Dotarem, $0.5 \mathrm{mmol} / \mathrm{ml}$; Guerbet BP, Paris, France), dynamic coronal sequences of the sellar region were acquired, followed by the acquisition of FSE T1-weighted images at a high resolution on the sagittal and coronal planes, both with a small field of view, targeted to the sellar region. For all high-resolution sequences, the slice thickness was $3 \mathrm{~mm}$. The MRI examination demonstrated an inhomogeneous mass (maximum diameter, $3.3 \mathrm{~cm}$ ) located in the intra- and suprasellar regions (Fig. 1). The solid and cystic components of the expansive mass cranially displaced the optic chiasm and partially obliterated the chiasmatic cistern.

Thereafter, the patient, immobilized by a thermoplastic mask, underwent computed tomography (CT) simulation (2 $\mathrm{mm}$ slice spacing) preliminary to radiotherapy. Image fusion between CT and T1-weighted MRI scans with contrast was performed on the basis of an anatomy surface-matching approach, a strategy aimed to match brain surfaces merging anatomical information, or landmark positions to establish the best correspondence. All clinical structures were delineated individually on the CT/MR axial slices by a radiologist (Dr Antonio Pierro) and a radiation oncologist (Dr Marica Ferro) subsequent to fusion approval by a senior RT physician (Professor Alessio G Morganti). The gross tumor volume was defined as the clinical target volume (CTV); to account for set-up uncertainties, a planning target volume (PTV) was generated from the CTV by a geometrical expansion of $5 \mathrm{~mm}$. Critical organs at risk (OAR), including the brainstem, chiasm, optical nerves and eyes, were contoured. A total dose of $63 \mathrm{~Gy}$ with $2.1 \mathrm{~Gy} /$ fraction was prescribed. The following maximum dose $\left(\mathrm{D}_{\max }\right)$ constraints were used: Lens, $<5$ Gy; eyeballs, <40 Gy; optic chiasm, <54 Gy; and brainstem, $<54$ Gy. A treatment plan was generated using the IMRT dose 

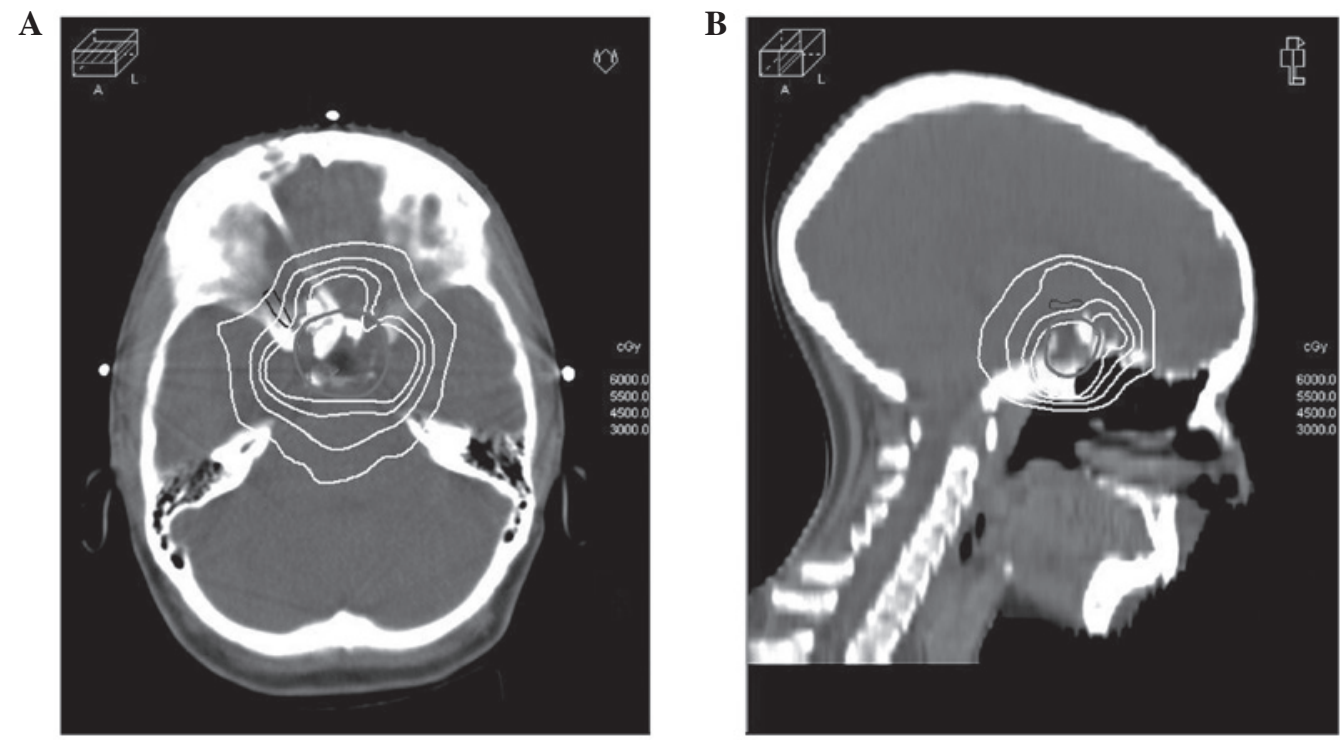

Figure 2. Dose distributions in (A) transverse and (B) sagittal planes obtained with intensity-modulated radiation therapy-based treatment planning.

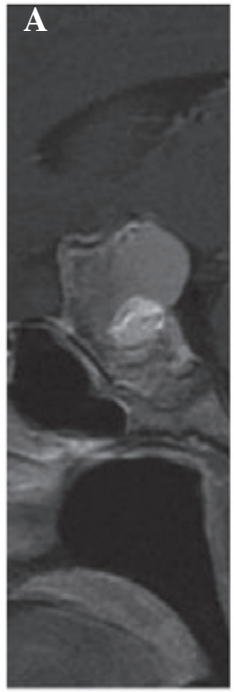

2008

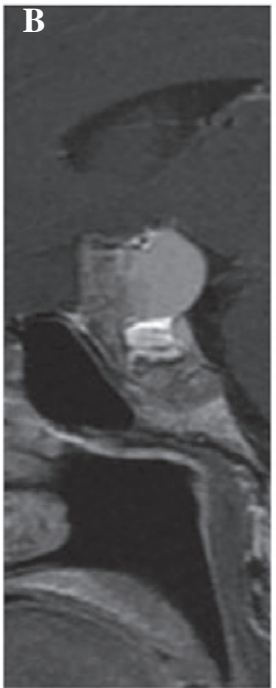

2009

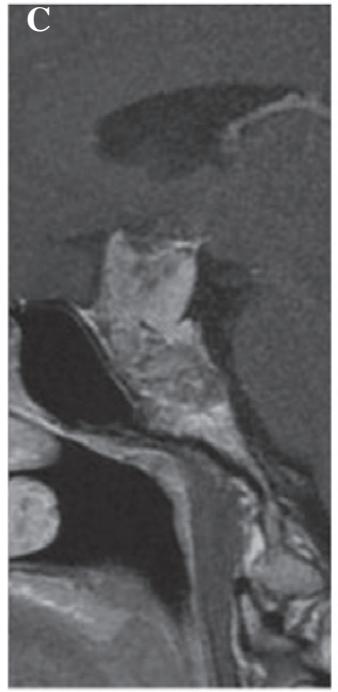

2010

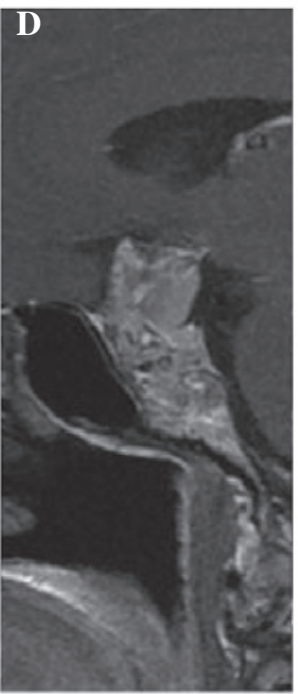

2011

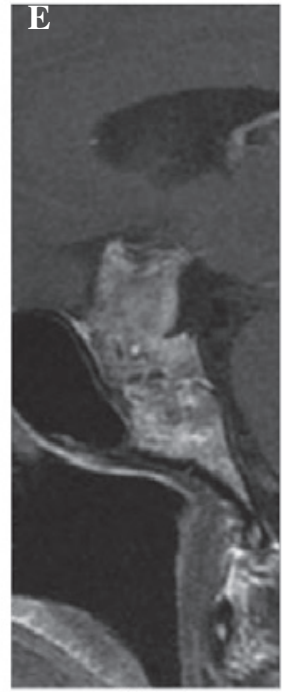

2014

Figure 3. Magnetic resonance images conducted: (A) prior to intensity-modulated radiation therapy; (B) in 2009, 1 year post-treatment; (C) in 2010, 2 years post-treatment, in which millimetric reduction in the size of the largest cystic component was observed; (D) in 2011, 3 years post-treatment, and (E) in 2014, 6 years post-treatment, in which a stability in terms of size and morphology was observed. Images are compared in the sagittal plane to clearly exhibit the tumor stability.

optimization engine in the Oncentra MasterPlan treatment planning system (Elekta, Crawley, UK). A class solution based on 7 fields (5 coplanar and 2 non-coplanar) was adopted, using an X-ray beam energy of 6-MV. Dose-volume histograms of the PTVs and OARs were analyzed. Target coverage was assessed by D5\% and D95\% metrics, which are the minimum doses delivered to 5 and $95 \%$ of the PTV volume, respectively. A uniformity index (UI) was used and defined as: UI=D5/D95. In addition, the mean dose and the percentage target volume receiving at least $90 \%$ of the prescribed dose (V90\%) were calculated, and the sparing of the OARs was evaluated by recording their maximum doses. Beams and fields set-up were iteratively optimized in order to maintain OAR doses below the critical values and obtain coverage of at least $90 \%$ of the prescribed dose up to $95 \%$ of the PTV volume. For the PTV,
D95\% and D5\% were 90.5 and $103.5 \%$ of the prescribed dose, respectively. The UI was 1.14 , PTV V95\% was $86.1 \%$ and the PTV mean dose was 62.1 Gy. Maximum doses to brainstem and optic chiasma were limited to $55.8 \mathrm{~Gy}$ (D1\%, $51.2 \mathrm{~Gy}$ ) and 54.1 Gy (D1\%, 53.5 Gy), respectively. Maximum doses to eyes, lens and optic nerves were limited to 18.4, 2.8 and 53.9 Gy, respectively. Fig. 2 illustrates the dose distribution in representative transverse and sagittal planes obtained with IMRT-based treatment planning, highlighting a highly shaped dose distribution and rapid periphery fall-off. The treatment was delivered using a Precise Treatment System linear accelerator, equipped with a standard 40-leaf dynamic multileaf collimator (Elekta AB, Stockholm, Sweden). The treatment time for each fraction was $\sim 14$ min and the number of monitor units was 580/fraction. A daily online correction protocol of 
isocenter position was applied using portal imaging (EPID I-VIEW; Elekta, Crawley, UK), with set-up correction in case of deviations $>0.3 \mathrm{~cm}$ in any direction (8). The treatment was conducted without interruption and without significant acute toxicity. At the subsequent follow-up evaluations, the patient presented patchy alopecia and reported occasional episodes of tinnitus and mild nausea. Neurological examinations were normal and clinical controls confirmed the absence of sequelae (such as headache, drowsiness, visual disturbances or hearing impairment) associated with the radiation treatment (3). In addition, the hormonal situation remained stable and the replacement therapy was unchanged. Following RT, the patient was checked by MRI once per year, according to the previously described protocol above. At 1 year subsequent to RT, no dimensional or morphological variations of the residual disease were observed, while 2 years later, a millimetric reduction in the size of the largest cystic component was noted. In all subsequent MRI scans, until 2014, the morphology and size of the mass remained unchanged, as shown in Fig. 3.

\section{Discussion}

In the present study, a patient suffering from a large $\mathrm{CP}$ residue following a third surgical resection was treated with a high IMRT dosage. Given the benign nature of the tumor and the requirement to reduce the risk of side effects, particularly due to the multiple surgical manipulations, a treatment plan providing the prescribed dose ( $63 \mathrm{~Gy}$ ) to a vast majority of the residual tumor was used, maintaining the lowest possible dose to the surrounding critical organs. At the 6-year follow-up, the patient presented no visual or neurological sequelae and did not require an increase in hormonal therapy. The $\mathrm{CP}$ remnants, following a small dimensional reduction, had remained stable.

The treatment of CP with radiation is particularly complex due to its proximity to other radiosensitive organs, which has stimulated the experimentation of advanced irradiation techniques. Boehling et al (9) evaluated the use of three-dimensional conformal proton RT (3D-PRT) and intensity-modulated proton therapy (IMPT) in pediatric patients with $\mathrm{CP}$, and compared these techniques with IMRT. The authors observed that the PTV target coverage was adequate for all modalities. IMRT and IMPT yielded the most conformal plans in comparison with 3D-PRT; however, the proton therapies were able to avoid excess integral radiation dose to a variety of normal structures at all dose levels, while maintaining equal target coverage (9). Gupta et al (7) treated 27 patients with residual, recurrent and/or progressive low-grade intracranial and skull base tumors with a 54-Gy total dose of tomotherapy. The early results (median follow-up, 19 months) of their study demonstrated the 2-year clinicoradiological progression-free survival and overall survival rates to be 93.3 and $100 \%$, respectively.

MRI is the examination of choice in the diagnosis of $\mathrm{CP}$ and the evaluation of spatial associations between tumors and adjacent structures $(2,10-12)$. In the present case, the combination of MRI and CT images allowed higher precision definition of the RT target and organs at risk. A further optimization may be based on the use of an adaptive strategy to take account of changes in PTV, as a result of cyst expansion, during treatment that might adversely affect tumor control rates and normal tissue. In fact, these variations can not be ignored, as documented by a study based on MRI monitoring throughout the course of RT that showed a target growth in the majority (64.3\%) of cases (13).

In conclusion, to the best of our knowledge, the current case is the first report of a high-dose ( $>60 \mathrm{~Gy}$ ) IMRT CP treatment with long active surveillance. The use of modern RT techniques, including IMRT and MRI image fusion, permitted the achievement of tumor stabilization without late radiation-induced sequelae.

\section{References}

1. Bunin GR, Surawicz TS, Witman PA, Preston-Martin S, Davis F, and Bruner JM: The descriptive epidemiology of craniopharyngioma. J Neurosurg 89: 547-551, 1998.

2. Curran JG and O'Connor E: Imaging of craniopharyngioma. Childs Nerv Syst 21: 635-639, 2005.

3. Winkfield KM, Bazan JG, Gibbs IC, Eng TY and Thomas CR: Nonmalignant disease. In: Perez and Brady's Principles and Practice of Radiation Oncology. Halperin EC, Perez CA and Brady LW (eds): 6th edition. Lippincott Williams \& Wilkins, Philadelphia, PA, USA, pp1729-1752, 2013.

4. Kalapurakal JA: Radiation therapy in the management of pediatric craniopharyngiomas - a review. Childs Nerv Syst 21: 808-816, 2005.

5. Rahmathulla G and Barnett GH: Minimally invasive management of adult craniopharyngiomas: An analysis of our series and review of literature. Surg Neurol Int 4 (Suppl 6): S411-S421, 2013.

6. Hader WJ, Steinbok P, Hukin J and Fryer C: Intratumoral therapy with bleomycin for cystic craniopharyngiomas in children. Pediatr Neurosurg 33: 211-218, 2000.

7. Gupta T, Wadasadawala T, Master Z,Phurailatpam R, Pai-Shetty R and Jalali R: Encouraging early clinical outcomes with helical tomotherapy-based image-guided intensity-modulated radiation therapy for residual, recurrent, and/or progressive benign/low-grade intracranial tumors: A comprehensive evaluation. Int J Radiat Oncol Biol Phys 82: 756-764, 2012.

8. Deodato F, Cilla S, Massaccesi M, Macchia G, Ippolito E, CaravattaL,Picardi V,RomanellaM,DiFalco C,Bartollino A, etal: Daily on-line set-up correction in 3D-conformal radiotherapy: Is it feasible? Tumori 98: 441-444, 2012.

9. Boehling NS, Grosshans DR, Bluett JB, Palmer MT, Song X, Amos RA, Sahoo N, Meyer JJ, Mahajan A and Woo SY: Dosimetric comparison of three-dimensional conformal proton radiotherapy, intensity-modulated proton therapy, and intensity-modulated radiotherapy for treatment of pediatric craniopharyngiomas. Int J Radiat Oncol Biol Phys 82: 643-652, 2012.

10. Tsuda M, Takahashi S, Higano S, Kurihara N, Ikeda H and Sakamoto K: CT and MR imaging of craniopharyngioma. Eur Radiol 7: 464-469, 1997.

11. Karavitaki N, Cudlip S, Adams CB and Wass JA: Craniopharyngiomas. Endocr Rev 27: 371-397, 2006.

12. Choi SH, Kwon BJ, Na DG, Kim JH, Han MH and Chang KH: Pituitary adenoma, craniopharyngioma, and Rathke cleft cyst involving both intrasellar and suprasellar regions: Differentiation using MRI. Clin Radiol 62: 453-462, 2007.

13. Beltran C, Roca M and Merchant TE: On the benefits and risks of proton therapy in pediatric craniopharyngioma. Int J Radiat Oncol Biol Phys 82: e281-e287, 2012. 